\title{
New insight on the far-UV SED and He II emission from low metallicity galaxies
}

\author{
Daniel Schaerer ${ }^{1,2}$ (D), Yuri Izotov ${ }^{3}$ and Tassos Fragos ${ }^{1}$ \\ ${ }^{1}$ Observatoire de Genève, Université de Genève, 51 Ch. des Maillettes, \\ 1290 Versoix, Switzerland \\ email: daniel.schaerer@unige.ch \\ ${ }^{2}$ CNRS, IRAP, 14 Avenue E. Belin, 31400 Toulouse, France \\ ${ }^{3}$ Bogolyubov Institute for Theoretical Physics, National Academy of Sciences of Ukraine, \\ 14-b Metrolohichna str., Kyiv, 03143, Ukraine
}

\begin{abstract}
Understanding the ionizing spectrum of low-metallicity galaxies is of great importance for modeling and interpreting emission line observations of early/distant galaxies.

Although a wide suite of stellar evolution, atmosphere, population synthesis, and photoionization models, taking many physical processes into account now exist, all models face a common problem: the inability to explain the presence of nebular He II emission, which is observed in many low metallicity galaxies, both in UV and optical spectra. Several possible explanations have been proposed in the literature, including Wolf-Rayet (WR) stars, binaries, very massive stars, X-ray sources, or shocks. However, none has so far been able to explain the major observations.

We briefly discuss the He II problem, available empirical data, and observed trends combining $\mathrm{X}$-ray, optical and other studies. We present a simple and consistent physical model showing that X-ray binaries could explain the long-standing nebular He II problem. Our model, described in Schaerer et al. (2019), successfully explains the observed trends and strength of nebular He II emission in large samples of low metallicity galaxies and in individual galaxies, which have been studied in detail and with multi-wavelength observations. Our results have in particular important implications for the interpretation of galaxy spectra in the early Universe, which will be obtained with upcoming and future facilities.
\end{abstract}

Keywords. galaxies: high-redshift, ultraviolet: galaxies, (ISM:) HII regions, X-rays: binaries

\section{The nebular He II problem}

Nebular He II $\lambda 4686$ emission in optical spectra of nearby sources has been discovered in the late 1980ies (see e.g. Pakull \& Angebault (1986); Garnett et al. (1991)). Except for planetary nebulae $(\mathrm{PN})$, where such emission is quite common, such objects are very rare in the local group, and the sources known then cover a diversity of objects, including a Wolf-Rayet star, a O type, a massive binary system, and one X-ray source (Garnett et al. (1991)). Since their discovery, the origin of this He II emission has been puzzling. Only stars with very high effective temperatures $T_{\text {eff }} \gtrsim 80-100 \mathrm{kK}$ emit non-negligible amounts of $\mathrm{He}^{+}$ionizing photons above $54 \mathrm{eV}$ (e.g. Schaerer (2002)), and because such temperatures are only reached in very peculiar evolutionary phases (e.g., in the WR or PN phase).

Zooming out of the local group, large numbers of star-forming galaxies showing nebular He II emission have been found, and these high-ionization lines are also present in AGN. E.g. using the Sloan survey, Shirazi \& Brinchmann (2012) have found 2865 galaxies with nebular He II. Excluding AGN, they have 200 star-forming galaxies, some of 


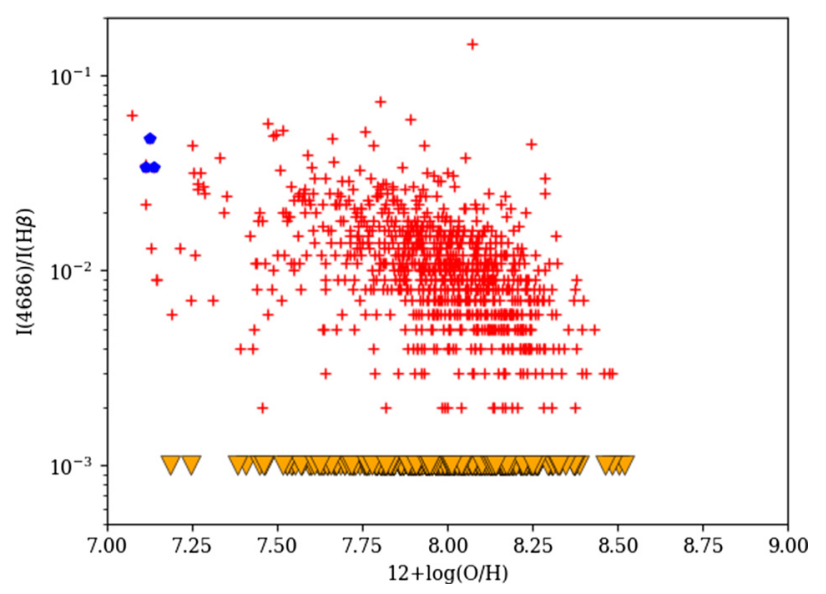

Figure 1. Observed $I(4686) / I(\mathrm{H} \beta)$ relative nebular line intensities as a function of metallicity. Observations of low-metallicity star-forming galaxies from the SDSS DR12-14 and observations of Izotov and collaborators are shown as red plusses. Measurements of the NW region of I Zw 18 are plotted with blue diamonds. The triangles show the non-detections $(\sim 1 / 3$ of the sources).

them showing also the presence of broad emission lines, indicative of WR stars, in these galaxies. Since "normal" stars should be the most numerous ones in star-forming regions/galaxies, and their UV/ionizing spectra are expected to be dominated by massive stars, very hot stars (cf. above) are at best very rare in these objects. Therefore no or very weak nebular He II is expected in general. In Figure 1 we show a compilation of more than 1400 star-forming galaxies or regions thereof showing nebular He II emission from Izotov et al. (2016). This sample reprensents a significant jump in statistics. They are selected based on the quality of the spectra, allowing direct metallicity determinations via the auroral line method. Active galaxies are excluded based on BPT diagrams.

Nebular He II emission traced by the UV He II $\lambda 1640$ line has also been observed in significant numbers of galaxies, some at low redshift and more at high- $z$ (e.g. Cassata et al. (2013), Berg et al. (2018), Nanayakkara et al. (2019)). The same trend of increasing frequency and strength of nebular He II $\lambda 1640$ with decreasing metallicity seems to be observed (Senchyna et al. (2017)), as for the optical line. This is expected since the He II lines are recombination lines from a simple hydrogenic atom, whose relative strengths between the UV and optical lines are to first order determined by atomic properties.

Since the discovery of He II emitters, other sources and processes that could emit more energetic photons than stellar sources have been suggested. These include X-ray binaries (XRBs), photoionization by X-rays, and strong shocks (see e.g. Pakull \& Angebault (1986); Garnett et al. (1991); Thuan \& Izotov (2005); Kehrig et al. (2015)). Other studies have explored if rotation and binarity could significantly alter the evolution of massive stars and create sufficiently hot stars and hence a harder ionizing spectrum (e.g. Szécsi et al. (2015), Götberg et al. (2018)). None of them has so far been able to quantitatively explain the observed intensity of the He II emission in low-metallicity galaxies. For example, the latest BPASS binary population and synthesis models underpredict the observed He II $\lambda 4686 / \mathrm{H} \beta$ intensities by $\sim$ one order of magnitude, as shown in Fig. 2 (right). Shocks and X-rays seem to be able to explain specific cases, but appear insufficient in others (see above references). However, no predictive model that would allow linking shock models to other galaxy properties exists, and especially it is unclear how shocks would reproduce 

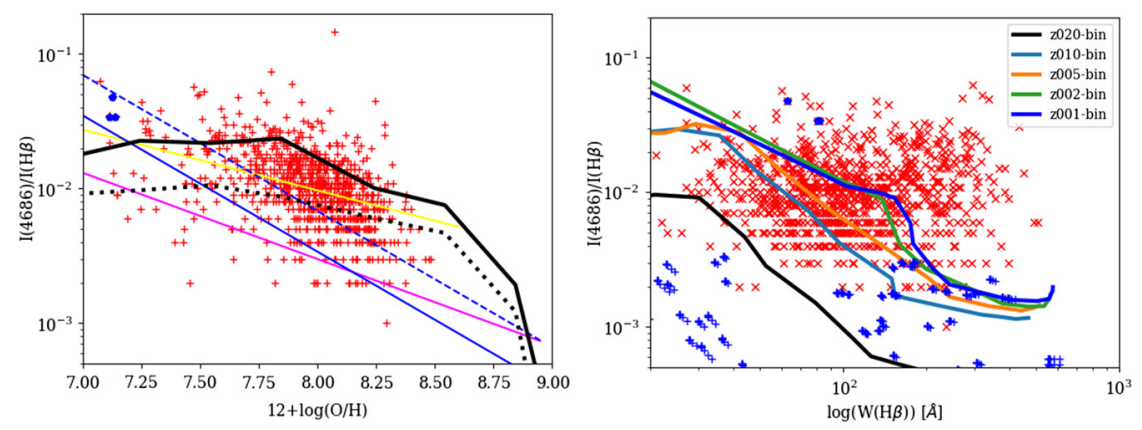

Figure 2. Observed and predicted $I(4686) / I(\mathrm{H} \beta)$ relative nebular line intensities as a function of metallicity (left) and the $\mathrm{H} \beta$ equivalent width (right) as an age indicator. Observations as in Fig. 1 Left: The observations are fitted by the yellow line (linear regression to the data points, detections only). Assuming $q=2 \times 10^{10}$ photon/erg, the empirical $L_{X} / \mathrm{SFR}-\mathrm{O} / \mathrm{H}$ relations of Douna et al. (2015) and Brorby et al. (2016) translate into He II intensities shown by the blue and magenta solid lines, respectively, assuming constant SFR. The black lines show the predicted He II intensity adopting $L_{X} /$ SFR predicted from the XRB synthesis models for a constant SFR over 10 Myr (dotted) and 0.1 Gyr (solid), and the same value of $q$. The blue dashed line differs from the solid line by assuming a value of $q$ that is a factor of two higher. Right: Instantaneous burst models for different metallicities, showing the predicted age dependence. Blue crosses show the BPASS models from Xiao et al. (2018). Figs. from Schaerer et al. (2019).

the trends of He II intensity with metallicity (Fig. 1). In short, the He II problem remains overall an unsolved problem calling for new/different approaches.

\section{A fresh look at the He II problem - empirical results}

The literature on X-ray emission in and from star-forming galaxies (SFGs) provides interesting insights, which could help understand the nature of He II emission. Indeed, the observations show overall the following picture (e.g. Mineo et al. (2012), Douna et al. (2015), Brorby et al. (2016)):

(a) First, the X-ray emission of SFGs is dominated by point-like sources, whose luminosity follows a power-law, including ultra-luminous X-ray sources (ULX) at the bright end $\left(L_{X}>10^{40} \mathrm{erg} \mathrm{s}^{-1}\right)$, high-mass X-ray binaries (HMXBs) and others.

(b) The total (spatially integrated) X-ray luminosity correlates to first order with the total star formation rate (SFR).

(c) There is an excess of X-ray emission per unit SFR, $L_{X} / \mathrm{SFR}$, at low metallicity.

(d) The observed scatter in $L_{X} /$ SFR is due to stochasticity of the ULX+HMXB at the high luminosity end of the X-ray luminosity function, which dominates the integrated X-ray emission.

These empirical findings suggest that X-ray bright sources (ULX and/or HMXB) could naturally explain the observed trends of He II emission, in particular the increase of $\mathrm{He}$ II $/ \mathrm{H} \beta$ with decreasing metallicity, for the following reasons and in the following way: First, the $\mathrm{H} \beta$ (or any other $\mathrm{H}$ recombination line) luminosity scales linearly with the SFR, i.e. $L(\mathrm{H} \beta) / S F R \approx$ const. Second, assuming that bright $\mathrm{X}$-ray sources emit a constant fraction of ionizing photons per X-ray luminosity above $54 \mathrm{eV}$, i.e. $q=Q\left(\mathrm{He}^{+}\right) / L_{X}$, the number of $\mathrm{He}^{+}$ionizing photons per $L_{X}$, is constant. Then the He II line luminosity is proportional to $L_{X}$, and the observed scaling of $L_{X}$ with SFR (point b) translates immediately to a relation between the $\mathrm{He}$ II $/ \mathrm{H} \beta$ line luminosities, hence also their relative intensity, which is the main observable. Finally, the observed increase of $L_{X} / \mathrm{SFR}$ towards low metallicity (point c) implies that $I(4686) / I(\mathrm{H} \beta)$ increases with decreasing $\mathrm{O} / \mathrm{H}$, as observed (Fig. 1). Furthermore, the observed scatter in $L_{X} /$ SFR (point d) would also 
lead to scatter in $I(4686) / I(\mathrm{H} \beta)$. This is in essence what could explain nebular He II at low metallicity, the observed metallicity trend, and possibly also the scatter, as we proposed in Schaerer et al. (2019).

To relate the observed trends of nebular He II with X-ray emission, we basically have to make only a single assumption, namely that He II emission originates from or is related to the bright sources which dominate in X-rays, i.e. ULX and/or HMBX. In fact, this assumption appears very reasonable and is supported by some empirical evidence. Indeed He II emission has been observed in several ULX and bright HMXB nebulae (e.g. Kaaret \& Corbel (2009), Pakull \& Mirioni (2012)). Furthermore such sources are present in SFGs, and they significantly modifiy the ionizing spectrum (and hence affect certain emission lines), as shown e.g. in the very detailed study of the metal-poor He II emitting galaxy I Zw 18 by Lebouteiller et al. (2017). Indeed in this galaxy, Chandra observations have shown the presence of a ULX and demonstrated that it is located in the main HiI region (NW region), where the bulk of He II emission originates from. This strongly supports a physical connection between bright X-ray sources and nebular He II. This finding is also confirmed by a new study of Heap et al. (2019), who also show that a well-motivated ULX spectral model is able to quantitatively explain the He II intensity in I Zw 18.

In Schaerer et al. (2019) we have used the observed X-ray and He II $\lambda 4686$ line luminosities of I Zw 18 to empirically derive $q=Q\left(\mathrm{He}^{+}\right) / L_{X}=(1.0-3.4) \times 10^{10}$ photon $/ \mathrm{erg}$ and adopt a "typical" value of $q=2 \times 10^{10}$ photon/erg. Predicted $I(4686) / I(\mathrm{H} \beta)$ relative intensities obtained using this simple approach are plotted in Fig. 2. They show that the observations can be reproduced approximately with these simple assumptions.

\section{Synthetic models including He II emission from X-ray binaries}

To go beyond the simple empirical attempt, we have combined X-ray binaries (XRB) population synthesis models with models describing spectral evolution of "normal" stellar populations. The main results, taken from Schaerer et al. (2019), are shown in Fig. 2.

Concretely, we have used models developed by Fragos et al. (2013ab) to study the cosmological evolution of XRB populations that were recently recalibrated to updated measurements of the cosmic star-formation history and metallicity evolution (Madau \& Fragos (2017). An important prediction of these models is the strong dependence of the XRB population on metallicity, both in terms of the formation efficiency of XRBs and the integrated X-ray luminosity of the whole population. This means that at low $\mathrm{O} / \mathrm{H}$ more XRBs are predicted and their X-ray luminosity is higher. Therefore these XRB models predict a higher $L_{X} / \mathrm{SFR}$ at low $\mathrm{O} / \mathrm{H}$, as shown by the observations discussed above (point c). Additionnaly, these models predict a strong dependence of $L_{X}$ on the age of the stellar population (see Schaerer et al. (2019)).

To bracket a range of star formation histories, we have examined instantaneous bursts and constant SFR. The predicted $I(4686) / I(\mathrm{H} \beta)$ intensities are shown in Fig. 2. They broadly cover the range of the observations, showing that our models can fairly well reproduce the observed $I(4686) / I(\mathrm{H} \beta)$, possibly with a superposition of populations.

As already mentioned, binary populations synthesis models such as BPASS do not predict sufficiently hard ionizing spectra to solve the He II problem (cf. Fig. 2). This shows that even hot/rejuvenated stars, which are created through binary interactions, are not sufficient, and that other sources (e.g. HMBX and ULX) need to be included. The earlier synthesis models of Cervinõ et al. (2002) including X-rays have also failed in this respect, since they primarily include soft X-ray emission from stellar winds, supernovae, and their remnants. 


\section{Conclusions and outlook}

Based on empirical X-ray data for star-forming galaxies showing a very similar behaviour of increasing $L_{X} / S F R$ as nebular He II emission with metallicity (Fig. 1), on the observational finding of a ULX in the strong He II $\lambda 4686$ emitting galaxy I Zw 18, and on the detailed modeling of this galaxy by Lebouteiller et al. (2017) and Heap et al. (2019), we have proposed that ULXs/HMXBs are the prime source of nebular He II emission low metallicity SFGs. A simple quantitative model using both empirical data and recent X-ray binary population synthesis models has recently been proposed by Schaerer et al. (2019) to solve the long-standing problem of nebular He II emission.

Obviously further applications, and tests are welcome and required, and this model does not exclude the contribution from other processes to the observed He II emission. For example, establishing observationally more firmly the correlation between X-ray and He II emission, both on spatially resolved scales and in integrated populations, should be a useful test. It also needs to be worked out if/how much the high-energy emission affects other emission lines, also in relation with the apparent hardening of the SEDs at high- $z$ inferred by several studies (see e.g. Stark (2016)). In any case observations show that X-rays are ubiquitous in star-forming galaxies (even if weaker than in AGN), as discussed amply in the literature (cf. Sect. 1). Therefore, their effects on the SEDs need to be examined, a task which should be more important for high- $z$ and low-metallicity galaxies, since HMXBs, ULXs, and their X-ray emission becomes stronger at low $\mathrm{O} / \mathrm{H}$.

\section{References}

Berg, D. A., Erb, D. K., Auger, M. W., Pettini, M., \& Brammer, G. B. 2018, ApJ, 859, 164

Brorby, M., Kaaret, P., Prestwich, A., \& Mirabel, I. F. 2016, MNRAS, 457, 4081

Cassata, P., Le Fèvre, O., Charlot, S., et al. 2013, A\& $A$, 556, A68

Cervinõ, M., Mas-Hesse, M., \& Kunth, D. 2002, A\&̈A, 392, 19

Douna, V. M., Pellizza, L. J., Mirabel, I. F., \& Pedrosa, S. E. 2015, A\&SA, 579, A44

Fragos, T., Lehmer, B., Tremmel, M., et al. 2013a, ApJ, 764, 41

Fragos, T., Lehmer, B. D., Naoz, S., Zezas, A., \& Basu-Zych, A. 2013b, ApJL, 776, L31

Garnett, D. R., Kennicutt, Jr., R. C., Chu, Y.-H., \& Skillman, E. D., 1991, ApJ, 373, 458

Götberg, Y., de Mink, S. E., Groh, J. H., et al. 2018, A\& A, 615, A78

Heap, S. R., Hubeny, I., Bouret, J.-C., \& Lanz, T. 2019, in Radiative signatures from the cosmos, ed. K. Werner \& T. Rauch, ASP Conference Series, in press

Izotov, Y. I., Guseva, N. G., Fricke, K. J., \& Henkel, C. 2016, MNRAS, 462, 4427

Kaaret, P. \& Corbel, S. 2019, ApJ, 697, 950

Kehrig, C., Vílchez, J. M., Pérez-Montero, E., et al. 2015, ApJL, 801, L28

Lebouteiller, V., Péquignot, D., Cormier, D., et al. 2017, A\&A, 602, A45

Madau, P. \& Fragos, T. 2017, ApJ, 840, 39

Mineo, S. et al. 2012, MNRAS, 419, 2095

Nanayakkara, T. et al. 2019, A\& A, 624, A89

Pakull, M. W. \& Angebault, L. P. 1986, Nature, 322, 511 EP

Pakull, M. \& Mirioni, L. 2012, arXiv:0202488

Schaerer, D. 2002, A\&A, 382, 28

Schaerer, D., Fragos, T., \& Izotov, Y. I. 2019, A\&A, 622, L10

Shirazi, M. \& Brinchmann, J. 2012, MNRAS, 421, 1043

Senchyna, P. et al. 2017, MNRAS, 472, 2608

Stanway, E. R. \& Eldridge, J. J. 2018, arXiv e-prints, 1811.03856

Stark, D. P. 2016, ARAA, 54, 761

Szécsi, D. et al. 2015, A\&\&A, 581, A15

Thuan, T. X. \& Izotov, Y. I. 2005, ApJS, 161, 240

Xiao, L., Stanway, E. R., \& Eldridge, J. J. 2018, MNRAS, 477, 904 